\title{
RADICALS AND POLYNOMIAL RINGS
}

\author{
K. I. BEIDAR, E. R. PUCZYŁOWSKI and R. WIEGANDT
}

(Received 16 February 2000; revised 3 January 2001)

Communicated by C. F. Miller

\begin{abstract}
We prove that polynomial rings in one indeterminate over nil rings are antiregular radical and uniformly strongly prime radical. These give some approximations of Köthe's problem. We also study the uniformly strongly prime and superprime radicals of polynomial rings in non-commuting indeterminates. Moreover, we show that the semi-uniformly strongly prime radical coincides with the uniformly strongly prime radical and that the class of semi-superprime rings is closed under taking finite subdirect sums.
\end{abstract}

2000 Mathematics subject classification: primary 16N20, 16N40, 16 N80.

\section{Introduction and preliminaries}

Köthe's Problem (is the sum of two nil left ideals nil?) is perhaps the most challenging problem in ring theory. It was posed in 1930 at the genesis of radical theory [5]. This problem has many equivalent formulations. One of the most interesting, which stimulated many further studies, is the following one due to Krempa [6]:

Does $R \in \mathscr{N}$ imply that the polynomial ring $R[x]$ in indeterminate $x$ over $R$ is in $\mathscr{J}$, where $\mathscr{N}$ and $\mathscr{J}$ denote the classes of nil rings and Jacobson radical rings, respectively?

In [11] it has been proved that $R \in \mathscr{N}$ implies $R[x] \in \mathscr{G}$, where $\mathscr{G}$ stands for the Brown-McCoy radical. This result can be viewed as an approximation of Köthe's Problem from above, because $\mathscr{J} \subset \mathscr{G}$. One can try to improve this approximation replacing $\mathscr{G}$ with some other radicals containing $\mathscr{N}$. Among the most natural radicals to consider are:

The second author was supported by KBN Grant 2 PO3A 03914 . The third author was supported by the Hungarian National Foundation for Scientific Research Grant \# T029525.

(c) 2002 Australian Mathematical Society 0263-6115/2002 \$A2.00+0.00 
- The Behrens radical $\mathscr{B}$. This is the upper radical determined by the class of rings possessing a non-zero idempotent.

- The antiregular radical $\mathscr{U} \nu$. This is the upper radical determined by the class $v$ of all von Neumann regular rings.

- The uniformly strongly prime radical $u$. A ring $R$ is said to be uniformly strongly prime, if there exists a finite subset $F$ of $R$, called a uniform insulator, such that $x F y \neq 0$ whenever $0 \neq x, y \in R$. The uniformly strongly prime radical is the upper radical determined by the class of uniformly strongly prime rings [8].

- The superprime radical. A ring $R$ is said to be (right) superprime [15] if every non-zero ideal $I$ of $R$ contains an element $a$ such that $r_{R}(a)=0$, where $r_{R}(a)$ denotes the right annihilator of $a$ in $R$. The superprime radical $\sigma$ is the upper radical determined by the class of all superprime rings.

The relations among these radicals are well known (see for instance [16]), and we summarize them in the following proposition.

PROPOSITION 1.1. $\mathscr{N} \subset \mathscr{J} \subset \mathscr{B} \subset \mathscr{G}, \mathscr{B} \subset \mathscr{U} \nu, \mathscr{N} \subset \sigma \subset u$. Moreover $\mathscr{G}\|\mathscr{U} v, \mathscr{G}\| u, \mathscr{U} v\|u, \mathscr{J}\| u$, where $\|$ stands for the relation 'not comparable'.

\section{COROLLARY 1.2. $\mathscr{B} \subset \mathscr{U} v \cap \mathscr{G} \subset \mathscr{G}$ and $\mathscr{U} \nu \cap \mathscr{G} \subset \mathscr{U} v$.}

PROOF. Let $V$ be a countably infinite dimensional space over the two element field $G F(2)$ and let $T$ be the ring of finite valued linear transformations of $V$. Further, let $t$ be the linear transformation of $V$ such that $t\left(e_{2 n}\right)=0$ and $t\left(e_{2 n-1}\right)=e_{2 n}$, $n=1,2, \ldots$, where $\left\{e_{1}, e_{2}, \ldots\right\}$ is a basis of $V$. Let $R$ be the subring of the ring of linear transformations of $V$ generated by $T \cup\{t\}$. It is not hard to check that $R$ is a subdirectly irreducible ring with heart equal to $T$ and $R / T$ is nilpotent. Since $T$ contains idempotents, $R \notin \mathscr{B}$. As $R / T$ is a non-zero nilpotent ring and $R$ is subdirectly irreducible, we conclude that $R \in \mathscr{U} \nu$. Clearly $R \in \mathscr{G}$. The rest follows immediately from Proposition 1.1.

The upper radical $\mathscr{N}_{s}$ determined by the class of rings which contain no non-zero nil left ideals or, equivalently, no non-zero nil right ideals is called the lower strong radical determined by $\mathscr{N}$. Clearly, $\mathscr{N} \subseteq \mathscr{N}_{s}$ and Köthe's problem is equivalent to the equality $\mathscr{N}=\mathscr{N}_{s}$. In this context it is natural to ask whether $\mathscr{N}_{s}$ behaves similarly to $\mathscr{N}$ when one takes polynomials. In [10, Corollary 3.3], it was observed that from the results of [11] it follows that if $L$ is a nil left ideal of a ring $R$, then $(L+L R)[x] \in \mathscr{G}$. This and the well-known fact (see for instance [7]) that the class $\{R \mid R[x] \in \mathscr{G}\}$ is radical easily imply that for every $\mathscr{N}_{s}$-radical ring $R, R[x] \in \mathscr{G}$. We shall show that the same holds if $\mathscr{G}$ is replaced by $\mathscr{U} v$ or $u$ (for $u$ we in fact get more, namely that polynomial rings in non-commuting indeterminates over rings in $\mathscr{N}_{s}$ are in $u$ ). 
We do not know whether for every nil ring $R$ the polynomial ring $R[x]$ belongs to $\sigma$. However we show that it does not hold for polynomial rings in sets of non-commuting indeterminates. We also answer some questions raised in [9] concerning $u$ and $\sigma$.

Given a ring $R, R^{*}$ will denote the ring obtained by adjoining an identity to $R$.

Throughout this paper $R[x]$ denotes the polynomial ring in an indeterminate $x$ over a ring $R$ and $R(X)$ denotes the ring of polynomials in non-commuting indeterminates from a set $X$. If $X=\{x\}$, then obviously $R\langle X\rangle=R[x]$.

\section{The antiregular radical}

The following theorem gives in particular an approximation of Köthe's problem by the antiregular radical.

THEOREM 2.1. For every $\mathscr{N}_{s}$-radical ring $R, R[x] \in \mathscr{U} \nu$.

PROOF. Note first that if $e$ is a right identity of a ring $A$, that is, $a e=a$ for every $a \in A$, then $r_{A}(e)$ is an ideal of $A$. Indeed, if $b \in r_{A}(e)$, then $A b=A e b=0$. Clearly, $a-e a \in r_{A}(e)$ for each $a \in A$. Hence $e+r_{A}(e)$ is an identity of $A / r_{A}(e)$. Thus $A$ can be mapped homomorphically onto a ring with an identity. Consequently, $A$ is not Brown-McCoy radical.

Suppose now that $R[x] \notin \mathscr{U} v$. Then there exists a surjective homomorphism $f: R[x] \rightarrow B$ such that $0 \neq B \in \nu$. Since von Neumann regular rings contain no non-zero nilpotent ideals, applying the Andrunakievich Lemma, one gets that $\operatorname{ker} f$ is an ideal of $R^{*}[x]$. Consequently, $(R \cap \operatorname{ker} f)[x] \subseteq \operatorname{ker} f$. Let $\bar{f}$ be the canonical homomorphism of $\bar{R}[x]$, where $\bar{R}=R /(R \cap \operatorname{ker} f)$, onto $B$ induced by $f$. Since $R \in \mathscr{N}_{s}$ and $\bar{R}$ is a non-zero homomorphic image of $R, \bar{R}$ contains a non-zero nil left ideal $L$. Obviously $\bar{f}(r) \neq 0$ for some $r \in L$. Since $B$ is von Neumann regular, there exists an idempotent $e \in B$ such that $B \bar{f}(r)=B e$. Now $\bar{f}((\bar{R} r)[x])=\bar{f}(\bar{R}[x] r)=\bar{f}(\bar{R}[x]) \bar{f}(r)=B \bar{f}(r)=B e$. Obviously $e$ is a right identity of $B e$, so $B e$ is not Brown-McCoy radical and consequently $(\bar{R} r)[x]$ is not Brown-McCoy radical. On the other hand, $\bar{R} r$ is a nil ring, so by [11, Corollary 3], the polynomial ring $(\bar{R} r)[x]$ is Brown-McCoy radical, a contradiction.

\section{The uniformly strongly prime radical}

THEOREM 3.1. (i) Given a set $X$, a ring $R$ is uniformly strongly prime if and only if the polynomial ring $R\langle X\rangle$ is uniformly strongly prime.

(ii) For every ring $R$ and every set $X, u(R\langle X\rangle)=u(R)\langle X\rangle$. 
ProOF. (i) Suppose that $R$ is a uniformly strongly prime ring with uniform insulator $F$. Let $a=\sum a_{i} m_{i}$ and $b=\sum b_{j} n_{j}$, where $a_{i}, b_{j} \in R$ and $m_{i}, n_{j}$ are monomials, be non-zero elements of $R\langle X\rangle$. Suppose that $m_{1}$ and $n_{1}$ are some of monomials of the least degree for which $a_{1} \neq 0$ and $b_{1} \neq 0$. Then $a_{1} F b_{1} \neq 0$, which easily implies that also $a F b \neq 0$. Hence $F$ is a uniform insulator for $R\langle X\rangle$.

Assume now that $R\langle X\rangle$ is uniformly strongly prime with a uniform insulator $G=\left\{g_{1}, \ldots, g_{k}\right\}$. Let $g_{1}=\sum r_{1 i} m_{1 i}, \ldots, g_{k}=\sum r_{k i} m_{k i}$, where $r_{j i} \in R$ and $m_{j i}$ are monomials. The set $F$ of all $r_{j i}$ is finite and it is clear that if $a F b=0$ for some $a, b \in R$, then also $a G b=0$. Since $G$ is a uniform insulator in $R\langle X\rangle$ we conclude that $a=0$ or $b=0$. Thus $F$ is a uniform insulator for $R$.

(ii) If $u(R)=0$, then $R$ is a subdirect sum of uniformly strongly prime rings $R_{i}$. Clearly $R\langle X\rangle$ is a subdirect sum of $R_{i}\langle X\rangle$. Hence by (i) we get that $u(R\langle X\rangle)=0$. It remains to prove that if $R$ is uniformly strongly prime radical, then so is $R\langle X\rangle$. Suppose that $R\langle X\rangle$ contains a proper ideal $I$ such that $R\langle X\rangle / I$ is uniformly strongly prime. Note that $I$ is also an ideal of $R^{*}\langle X\rangle$. Let $\phi$ be the canonical homomorphism of $R^{*}\langle X\rangle$ onto $R^{*}\langle X\rangle / I$. Clearly $R\langle X\rangle / I$ is generated by $\phi(R) \phi(T)$, where $T$ is the free monoid generated by $X$. Let $F=\left\{f_{1}, \ldots, f_{n}\right\}$ be a uniform insulator of $R\langle X\rangle / 1$. Each $f_{i}$ is a finite sum of elements of the form $a_{i j} m_{i j}$, where $a_{i j} \in \phi(R)$ and $m_{i j} \in \phi(T)$. It is clear that the set of all $a_{i j}$ is a uniform insulator of $\phi(R)$, so $\phi(R)$ is uniformly strongly prime. This contradicts the assumption that $R$ is uniformly strongly prime radical.

PROPOSITION 3.2. Suppose that $S$ is a multiplicative semigroup with 0 . If $S$ is nil, then for every finite (non-empty) subset $F$ of $S$ and every $a \in S$ for which $a F a \neq 0$ there exists $b \in S$ such that $a b a \neq 0$ and $|a b a F a b a|<|a F a|$.

ProOF. Suppose that $F=\left\{x_{1}, \ldots, x_{n}\right\}$ and $a F a \neq 0$. We can assume that $a x_{1} a \neq 0$. Let $k$ be a natural number such that $\left(a x_{1}\right)^{k} \neq 0$ but $\left(a x_{1}\right)^{k+1}=0$. If $\left(a x_{1}\right)^{k} a \neq 0$, then for $b=x_{1}\left(a x_{1}\right)^{k-1}$ (for $k=1$ we take $\left.b=x_{1}\right), a b a=\left(a x_{1}\right)^{k} a \neq 0$ and $a b a F a b a \subseteq a b a\left\{x_{2}, \ldots, x_{n}\right\} a b a$, so we are done. If $\left(a x_{1}\right)^{k} a=0$, then $k \geq 2$ and for $b=x_{1}\left(a x_{1}\right)^{k-2}, a b a \neq 0$ and $a b a x_{1} a b a=\left(a x_{1}\right)^{k} a b a=0$. Hence again $|a b a F a b a|<|a F a|$. The result follows.

COROLLARY 3.3. (i) If $S$ is a non-zero nil semigroup with 0 , then for every finite subset $F$ of $S$ there is a non-zero $a \in S$ such that $a F a=0$.

(ii) If $R$ is a ring generated by a nil subsemigroup $S$ of the multiplicative semigroup of $R$, then $R \in u$.

Proof. Statement (i) is a direct consequence of Proposition 3.2.

(ii) Let $\phi$ be a ring homomorphism of $R$ onto $R^{\prime}$. Obviously $R^{\prime}$ is generated by $\phi(S)$. Take any $r_{1}, \ldots, r_{n} \in R^{\prime}$. For every $1 \leq i \leq n$ there is a finite set $\left\{s_{i j}\right\} \subseteq \phi(S)$ 
and integers $e_{i j}$ such that $r_{i}=\sum_{j} e_{i j} s_{i j}$. Since the semigroup $\phi(S)$ is nil, by (i), $\phi(S)=0$ or there is $0 \neq a \in \phi(S)$ such that $a s_{i j} a=0$ for all $i, j$. Hence $a r_{i} a=0$ for all $1 \leq i \leq n$. This shows that $R^{\prime}$ is not uniformly strongly prime. Consequently, $R \in u$.

Clearly Corollary 3.3 (ii) implies that $\mathscr{N} \subseteq u$ (see for example [14]). In fact it gives more.

COROLLARY 3.4. $\mathscr{N}_{s} \subseteq u$.

PROOF. Obviously it suffices to prove that if a ring $R$ has a non-zero nil left ideal $L$, then $u(R) \neq 0$. Note that $\left\{l r \mid l \in L, r \in R^{*}\right\}$ is a nil subsemigroup of the multiplicative semigroup of $R$ generating $L R^{*}$ as a ring. Hence by Corollary 3.3 (ii), $L R^{*} \in u$. Consequently, $0 \neq L R^{*} \subseteq u(R)$.

Theorem 2.1, Theorem 3.1 and Proposition 1.1 along with the quoted result of [11] yield

COROLlaRY 3.5. If $R \in \mathscr{N}$, then $R[x] \in \mathscr{U} v \cap \mathscr{G} \cap u$. The positive solution of Köthe's Problem would imply that $R[x] \in \mathscr{J} \cap u$ for every nil ring $R$.

Thus Corollary 3.5 provides also another equivalent formulation of Köthe's Problem: Does $R \in \mathscr{N}$ imply $R[x] \in \mathscr{J} \cap u$ ?

The position of the radical classes discussed so far is given in the following diagram:

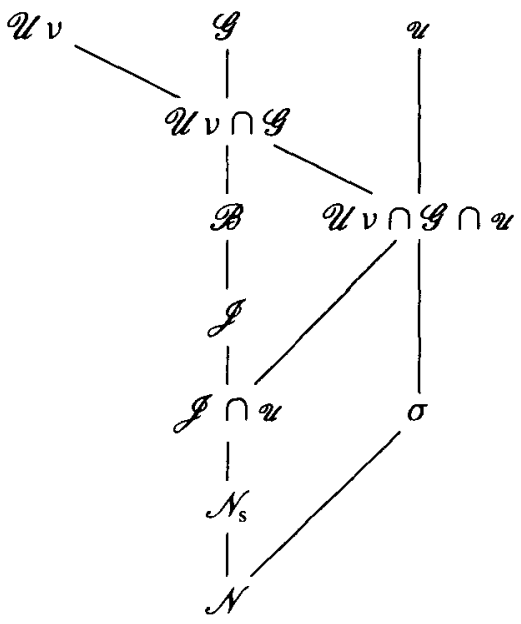

Olson, Le Roux and Heyman [9] defined a ring $R$ to be semi-uniformly strongly prime if every non-zero ideal $I$ of $R$ contains a finite subset $F$, called an insulator of 
$I$, such that for every $0 \neq i \in I, i F i \neq 0$. They proved that the class of all semiuniformly strongly prime rings is weakly special. The upper radical $u^{\prime}$ determined by this class could be potentially another radical to examine when approximating Köthe's Problem. However, as we shall show, it coincides with the uniformly strongly prime radical. This answers a question raised in [9].

We shall need the following two lemmas proved by Handelman in [3].

LEMMA 3.6 ([3, Lemma 7]). If a semiprime ring $R$ contains no infinite direct sums of non-zero ideals, then $R$ satisfies the ascending and descending conditions on annihilators of ideals.

LEMMA 3.7 ([3, Lemma 8]). If $I$ is a non-zero ideal in a semiprime ring $R$ and the annihilator $\bar{I}$ of $I$ is maximal among annihilators of ideals in $R$, then $R / \bar{I}$ is a prime ring.

PROPOSITION 3.8. The radical $u^{\prime}$ coincides with the uniformly strongly prime radical $u$.

PROOF. It suffices to prove that every semi-uniformly strongly prime ring $R$ can be homomorphically mapped onto a non-zero uniformly strongly prime ring. Clearly $R$ is semiprime and contains no infinite direct sum of non-zero ideals. Hence applying Lemma 3.6 and Lemma 3.7 we obtain that $R$ contains a non-zero ideal $K$ such that $R / \bar{K}$ is a prime ring. We claim that $R / \bar{K}$ is uniformly strongly prime. Let $F$ be an insulator of $K$. We shall prove that $F+\bar{K}$ is a uniform insulator of $R / \bar{K}$. Suppose that $x, y \in R \backslash \bar{K}$ and $x F y \subseteq \bar{K}$. Let $P=\{r \in R \mid x F r \subseteq \bar{K}\}$ and $L=\{r \in R \mid r F P \subseteq \bar{K}\}$. Clearly $P$ and $L$ are right and left ideals of $R$, respectively, and both of them strictly contain $\bar{K}$. Hence $0 \neq K L \subseteq K \cap L$ and $0 \neq P K \subseteq P \cap K$, so $P \cap K$ and $L \cap K$ are non-zero right and left ideals of $K$, respectively. Since $R / \bar{K}$ is a prime ring and $K$ is isomorphic to an ideal of that ring, $K$ is a prime ring. Consequently, $0 \neq(P \cap K)(L \cap K) \subseteq L \cap P \cap K$. Now for every $0 \neq t \in L \cap P \cap K$, $t F t=0$. Hence $F$ is not an insulator of $K$, a contradiction.

\section{The superprime radical}

Clearly $\mathscr{N} \subseteq \sigma$ but we do not know whether $\mathscr{N}_{s} \subseteq \sigma$. We also do not know whether if $R \in \mathscr{N}$, then $R[x] \in \sigma$. This would improve the approximation of Köthe's Problem. It is not hard to check that for every ring $R$ the ring $M(R)$ of countable matrices over $R$ which have only finitely many non-zero entries is in $\sigma$, and if $R \in \mathscr{N}_{s}$ then also $M(R) \in \mathscr{N}_{s}$. In particular, if $R \in \mathscr{N}$ then $M(R) \in \mathscr{N}_{s}$. The problem whether for every $R \in \mathscr{N}$ also $M(R) \in \mathscr{N}$ is equivalent to Köthe's problem 
$([6,13])$. It is clear that if $R$ is locally nilpotent, then $M(R) \in \mathscr{N}$. There also exist not locally nilpotent rings $R$ such that $M(R) \in \mathscr{N}$. As it was noted in [7] and in [1, Lemma 59] if $R$ is Golod's example of [2] (recall that this is an example of a nil ring which is not locally nilpotent), then $R[x] \in \mathscr{N}$, which implies that $M(R) \in \mathscr{N}$. An example of a ring $R$ for which $M(R)$ is nil and $R[x]$ is not nil was constructed in [12]. Note that for every ring $R$ and every set $X, M(R)\langle X\rangle \simeq M(R\langle X\rangle) \in \sigma$. These show that there are many nil rings $R$ such that for every set $X, R\langle X\rangle \in \sigma$. We shall show that there are also nil rings for which it does not hold.

A ring $R$ is called (right) strongly prime if every non-zero ideal of $R$ contains a finite subset $F$ such that the right annihilator $r_{R}(F)$ of $F$ in $R$ is equal to zero. Obviously every strongly prime ring is prime and every superprime ring is strongly prime.

If $R$ is a finitely generated non-nilpotent ring, then applying Zorn's lemma one can find in $R$ an ideal maximal with respect to the property that $R^{n} \nsubseteq I$ for all $n=1,2, \ldots$ (Zorn's lemma applies because all $R^{n}$ are finitely generated rings). Obviously $R / I$ is a prime ring. For every ideal $J$ of $R$ strictly containing $I$ there is a natural number $n$ such that $R^{n} \subseteq J$. The ring $R^{n}$ is generated by a finite set, say, $F$. Clearly for arbitrary $x \in R, F x \subseteq I$ if and only if $R^{n} x \subseteq I$. This easily implies that $R / I$ is strongly prime.

There are finitely generated non-nilpotent nil rings. Hence the foregoing remark implies that there exist strongly prime nil rings.

Now we shall prove

THEOREM 4.1. If $R$ is a strongly prime ring, then for every set $X$ with $|X| \geq 2$, the ring $R\langle X\rangle$ is superprime.

Proof. Let $I$ be a non-zero ideal in $R^{*}\langle X\rangle$ contained in $R\langle X\rangle$ and let $m$ be a monomial of least degree such that for some $0 \neq r \in R, r_{1}, \ldots, r_{n} \in R$ and some monomials $m_{1}, \ldots, m_{n}, r m+r_{1} m_{1}+\cdots+r_{n} m_{n} \in I$. The set $J$ consisting of all coefficients of $m$ in elements belonging to $I$ is a non-zero ideal of $R^{*}$. Since $R$ is strongly prime there are elements $a_{1}, \ldots, a_{t} \in J$ such that $r_{R}\left(\left\{a_{1}, \ldots, a_{t}\right\}\right)=0$. Suppose that they appear as coefficients of $m$ in polynomials $f_{1}, \ldots, f_{t} \in I$. Let $x$ and $y$ be two distinct elements in $X$. Then $f=f_{1} x y^{t}+f_{2} x^{2} y^{t-1}+\cdots+f_{t} x^{t} y \in I$. We claim that $r_{R(X)}(f)=0$. Indeed, if for some $g \in R\langle X\rangle, f g=0$, then $\bar{f} \bar{g}=0$, where $\bar{f}$ and $\bar{g}$ are the least components of $f$ and $g$ with respect to the gradation of $R\langle X\rangle$ given by the degree. Suppose that $\bar{f}=b_{1} p_{1}+\cdots+b_{l} p_{l}$, where $b_{i} \in R$ and $p_{i}$ are distinct monomials and similarly $\bar{g}=c_{1} q_{1}+\cdots+c_{s} q_{s}$. Note that $\left\{a_{1}, \ldots, a_{t}\right\} \subseteq\left\{b_{1}, \ldots, b_{l}\right\}$. Moreover $p_{i} q_{j}=p_{u} q_{w}$ if and only if $p_{i}=p_{u}$ and $q_{j}=q_{w}$. This shows that $b_{i} c_{j}=0$ for all $i, j$. Consequently, $c_{j} \in r_{R}\left(\left\{a_{1}, \ldots, a_{t}\right\}\right)=0$ and $\bar{g}=0$, a contradiction. Now it suffices to apply the Andrunakievich lemma to get that every non-zero ideal of $R\langle X\rangle$ 
contains an element $i$ such that $r_{R\langle X\rangle}(i)=0$. This proves that $R\langle X\rangle$ is superprime.

Olson, Re Roux and Heyman [9] defined a ring $R$ to be (right) semi-superprime if for every non-zero ideal $I$ of $R$ there exists $i \in I$ such that $r_{l}(i)=0$. They proved that all semi-superprime rings are finite subdirect sums of superprime rings and asked whether the converse holds. We shall show that it is indeed the case. Since all superprime rings are semi-superprime it suffices to prove the following

THEOREM 4.2. Every ring which is a subdirect sum of two semi-superprime rings is semi-superprime.

PROOF. It is clear that every semi-superprime ring $R$ contains no infinite direct sum of non-zero ideals. Note that ideals of $R$ are precisely $R \otimes R^{o p}$-submodules of $R$. Hence the Goldie dimension of $R$ as $R \otimes R^{o p}$-module is finite. Thus a direct sum $R_{1} \oplus \cdots \oplus R_{n}$ of uniform $R \otimes R^{o p}$-submodules of $R$ is an essential $R \otimes R^{o p_{-}}$ submodule of $R$. Since the class of semi-superprime rings is hereditary and consists of semiprime rings, an ideal $I$ of $R$ is uniform as an $R \otimes R^{o p}$-module if and only if $I$ is a prime ring. Clearly prime semi-superprime rings are superprime. Consequently, every semi-superprime ring $R$ contains a direct sum $I_{1} \oplus \cdots \oplus I_{n}$ of ideals $I_{i}$ which are superprime rings and such that $I_{1} \oplus \cdots \oplus I_{n}$ 'is an essential ideal of $R$.

Suppose now that $R$ is a subdirect sum of two semi-superprime rings, that is, $R$ contains ideals $I, J$ such that $I \cap J=0$ and $R / I$ and $R / J$ are semi-superprime rings. Since $I$ is isomorphic to an ideal in $R / J$ and $R / J$ is semi-superprime, $I$ contains an essential direct sum $I_{1} \oplus \cdots \oplus I_{n}$ of ideals which are superprime rings. Applying the Andrunakievich lemma it is not hard to show that all $I_{i}$ can be chosen to be ideals of $R$. Note that if $I_{n+1}, \ldots, I_{t}$ are non-zero ideals in $R$ such that the sum $I_{1}+\cdots+I_{n}+I_{n+1}+\cdots+I_{t}$ is direct, then $\left(I+I_{n+1}\right) / I+\cdots+\left(I+I_{t}\right) / I$ is a direct sum of non-zero ideals of $R / I$. Thus $t-n$ does not exceed the Goldie dimension of the $(R / I) \otimes(R / I)^{o p}$-module $R / I$. Consequently, we can assume that all $I_{i}$ are superprime rings and $I_{1} \oplus \cdots \oplus I_{t}$ is an essential ideal of $R$. Note that if $M$ is an ideal of $I_{1} \oplus \cdots \oplus I_{i}$, then $M \cap I_{i} \neq 0$ if and only if $\pi_{i}(M) \neq 0$, where $\pi_{i}$ is the natural projection of $I_{1} \oplus \cdots \oplus I_{t}$ onto $I_{i}$. If $T$ is a non-zero ideal in $I_{1} \oplus \cdots \oplus I_{t}$, then $\left(T \cap I_{1}\right) \oplus \cdots \oplus\left(T \cap I_{t}\right)$ is an essential ideal in $T$. Indeed, if $M \subseteq T$ is a non-zero ideal of $I_{1} \oplus \cdots \oplus I_{t}$, then $\pi_{i}(M) \neq 0$ for some $i$, so $0 \neq M \cap I_{i} \subseteq T \cap I_{i}$. Take now any non-zero ideal $K$ in $R$ and put $T=K \cap\left(I_{1} \oplus \cdots \oplus I_{t}\right)$. Since $I_{1} \oplus \cdots \oplus I_{t}$ is essential in $R$ and $R$ is semiprime, $T$ is essential in $K$. Note that since all $l_{i}$ are superprime, for every $i$ for which $T \cap I_{i} \neq 0$ there is an $a_{i} \in T \cap I_{i}$ such that $r_{l_{i}}\left(a_{i}\right)=0$. Then for $a=\sum a_{i}, r_{U}(a)=0$, where $U=\left(T \cap I_{1}\right) \oplus \cdots \oplus\left(T \cap I_{t}\right)$. However $U$ is essential in $T$ and $T$ is essential in $K$, so $U$ is essential in $K$. Now $U \cap r_{K}(a)=r_{U}(a)=0$, so $r_{K}(a) U=0$. Hence, since $U$ is semiprime and essential in $K, r_{K}(a)=0$. The result follows. 


\section{References}

[1] G. M. Bergman, 'Radicals, tensor products, and algebraicity', Israel Math. Conf. Proc. 1 (1989), 150-192.

[2] E. S. Golod, 'On nil algebras and finitely approximable $p$-groups', Izv. Akad. Nauk SSSR Ser. Mat. 28 (1964), 273-276 (Russian).

[3] D. Handelman, 'Strongly semiprime rings', Pacific J. Math. 60 (1975), 115-122.

[4] D. Handelman and J. Lawrence, 'Strongly prime rings', Trans. Amer. Math. Soc. 211 (1975), 209-223.

[5] G. Köthe, 'Die Struktur der Ringe, deren Restklassenring nach dem Radikal vollständing reduzibel ist', Math. Z. 32 (1930), 161-186.

[6] J. Krempa, 'Logical connections among some open problems in non-commutative rings', Fund. Math. 76 (1972), 121-130.

[7] —_. 'Radicals of semigroup rings', Fund. Math. 85 (1974), 57-71.

[8] D. M. Olson, 'A uniformly strongly prime radical', J. Austral. Math. Soc. (Series A) 43 (1987), 95-102.

[9] D. M. Olson, H. J. Le Roux and G. A. P. Heyman, 'Classes of strongly semiprime rings', in: Theory of radicals, Colloq. Math. Soc. János Bolyai 61 (North-Holland, Amsterdam, 1993) pp. 197-208.

[10] E. R. Puczyłowski, 'Some results and questions on nil rings', Mat. Contemp. 16 (1999), 265-280.

[11] E. R. Puczyłowski and A. Smoktunowicz, 'On maximal ideals and the Brown-McCoy radical of polynomial rings', Comm. Algebra 26 (1998), 2473-2482.

[12] — , 'On Amitsur's conjecture', Preprint P99-05, (Institute of Mathematics, Warsaw University, 1999).

[13] A. D. Sands, 'Radicals and Morita contexts', J. Algebra 24 (1973), 335-345.

[14] J. E. van den Berg, 'On uniformly strongly prime rings', Math. Japonica 38 (1993), 1157-1166.

[15] S. Veldsman, 'The superprime radical', in: Proceedings of the Krems Conference (August 16-23, 1985), Contrib. general algebra 4 (Hölder-Pichler-Tempsky, Wien and B. G. Teubner, Stuttgart, 1987) pp. 181-188.

[16] R. Wiegandt, 'Rings distinctive in radical theory', Quaestiones Math. 23-24 (1999), 447-472.

Department of Mathematics

National Cheng-Kung University

Tainan

Taiwan

e-mail: beidar@mail.ncku.edu.tw
Institute of Mathematics

University of Warsaw

Warsaw

Poland

e-mail: edmundp@mimuw.edu.pl

Institute of Mathematics

Hungarian Academy of Sciences

Budapest

Hungary

e-mail: wiegandt@math-inst.hu 
